R. A. Herb

Nagoya Math. J.

Vol. 64 (1976), 47-61

\title{
GHARACTER FORMULAS FOR DISCRETE SERIES ON SEMISIMPLE LIE GROUPS
}

\author{
REBECCA A. HERB*
}

\section{§1. Introduction}

Let $G$ be a connected, semisimple real Lie group with finite center, $K$ a maximal compact subgroup of $G$. Assume $\operatorname{rank} G=\operatorname{rank} K$. Let (S) be the Lie algebra of $G$, $\mathbb{S}_{C}$ its complexification. If $G_{C}$ is the simply connected complex analytic group corresponding to $\mathbb{B}_{C}$, assume $G$ is the real analytic subgroup of $G_{C}$ corresponding to (s).

In this case, $G$ always has discrete series representations. The characters of these representations are distributions on the group $G$, realizable as locally integrable functions. Formulas for these characters are known up to certain integer constants which have only been evaluated for a few special cases. The purpose of this paper is to give information on how these constants can be computed in general, and to illustrate the method for several new cases.

For any Cartan subalgebra $\mathfrak{h}$ of $\mathbb{S}$, let $\mathfrak{h}_{c}$ denote its complexification, $\Phi\left(\mathscr{\mho}_{c}, \mathfrak{h}_{c}\right)$ the set of roots of the pair $\left(\mathscr{S}_{c}, \mathfrak{h}_{c}\right)$, and $W\left(\mathscr{\mho}_{c}, \mathfrak{h}_{c}\right)$ the Weyl group generated by the reflections corresponding to the roots. Let $\pi^{\mathfrak{b}}(H)$ $=\prod \alpha(H)$, the product over all $\alpha$ in $\Phi^{+}\left(\mho_{C}, \mathfrak{h}_{C}\right), H$ any element of $\mathfrak{h}$.

Denote by $\mathfrak{f}$ the subalgebra of $\mathbb{S}$ corresponding to $K$, and let $t$ be a Cartan subalgebra of $\mathbb{E}$ such that $t \subseteq \mathfrak{H}$. Consider the space $\mathscr{F}$ of all pure imaginary linear functions on $t$. Let $\mathscr{F}^{\prime}=\{\lambda \in \mathscr{F}:\langle\lambda, \alpha\rangle \neq 0$ for all $\left.\alpha \in \Phi\left(\mho_{c}, t_{c}\right)\right\}$, the regular elements of $\mathscr{F}$. Then for each $\lambda \in \mathscr{F}^{\prime}$ there exists a unique invariant distribution $T_{\lambda}$ on $\&$ characterized by certain properties [2a), p. 277].

Let $W_{K}$ be the subgroup of $W\left(\mathbb{S}_{c}, \mathrm{t}_{c}\right)$ generated by reflections cor-

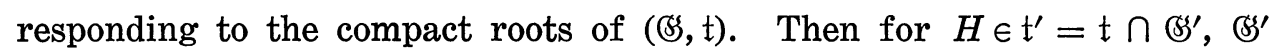
the set of regular elements of (S),

Received July 15, 1975.

* Supported in part by NSF grant MP572-05055 A02. 


$$
T_{\lambda}(H)=\pi^{\mathrm{t}}(H)^{-1} \sum_{w \in W_{K}} \operatorname{det} w \exp (w \lambda(H)) .
$$

Given any Cartan subalgebra $\mathfrak{h}$ of $(S)$ there exists $y \in G_{C}$ such that $y\left(\mathfrak{t}_{c}\right)=\mathfrak{h}_{c}$. Then for any connected component $\mathfrak{h}^{+}$of $\mathfrak{h}^{\prime}(R)=\{H \in \mathfrak{h}: \alpha(H)$ $\neq 0$ for all real roots $\left.\alpha \in \Phi\left(\mathscr{S}_{c}, \mathfrak{h}_{c}\right)\right\}$, there are integers $c_{y}\left(w: \lambda: \mathfrak{h}^{+}\right)$such that for $H \in \mathfrak{h}^{+} \cap \mathfrak{h}^{\prime}, \mathfrak{h}^{\prime}=\mathfrak{h} \cap \mathbb{B S}^{\prime}$,

$$
T_{\lambda}(H)=\pi^{\mathfrak{h}}(H)^{-1} \sum_{w \in W\left(\mathbb{S}_{C}, \mathfrak{t} c^{)}\right.} \operatorname{det} w c_{y}\left(w: \lambda: \mathfrak{h}^{+}\right) \exp \left({ }^{y}(w \lambda)(H)\right) .
$$

In $\S 2$ of this paper we outline an inductive procedure by which these integers can be computed, and in $\S 3$ illustrate the method by explicit computations for the first two stages of the induction in the general case, and by giving the complete solution for the case where (s) has exactly $n+1$ conjugacy classes of Cartan subalgebras, $n=\operatorname{rank}(G / K)$.

The integers have been computed by Harish-Chandra for the case $\operatorname{rank}(G / K)=1([2 b)])$ and by $H$. Ferguson for the case where $\varangle_{c}$ is the simple complex Lie algebra with root system of type $G_{2}$ ([1]). The method given in this paper is different from that used by Harish-Chandra for $\operatorname{rank}(G / K)=1$ (although it relies heavily on his work in [2a)] and [2b)]), and is an extension of the method used by Ferguson. Hirai has computed the integers for the groups $S U(p, q)$ and $S p(2, R)$ using specific matrix computations.

The unitary character group $\hat{T}$ of $T, T$ the Cartan subgroup of $G$ corresponding to $t$, may be identified with a lattice, $L_{T}$, in $\mathscr{F}$. To each $\lambda \in L_{T}$ is associated a central eigendistribution $\Theta_{\lambda}$ on $G$ ([2a), p. 289], [2b), p. 90]). If $\lambda \in L_{T}^{\prime}=L_{T} \cap \mathscr{F}^{\prime}$, a constant multiple of $\Theta_{\lambda}$ is the character of a discrete series representation of $G$, and all discrete series characters are of this form.

To explicitly describe the $\Theta_{\lambda}$ it is necessary to evaluate certain constants which are directly related to the integers $c_{y}\left(w: \lambda: \mathfrak{h}^{+}\right)$. In $\S 4$, using the results of $\S 3$, we give explicit formulas for the $\Theta_{\lambda}$ on the Cartan subgroups of $G$ having vector part of dimension one or two. The Cartan subgroups in the dimension one case are those corresponding to maximal parabolic subgroups of $G$. The results for dimension two give a complete solution for the case $\operatorname{rank}(G / K)=2$. We also give complete formulas in the case where $G$ has exactly $n+1$ conjugacy classes of Cartan subgroups, $n=\operatorname{rank}(G / K)$. 


\section{§2. The Constants on \&3}

We retain the notation of the introduction. Since $T_{2}$ is an invariant distribution, it suffices to determine the integers $c_{y}\left(w: \lambda: \mathfrak{h}^{+}\right)$for one representative of each conjugacy class of Cartan subalgebras. Let $\theta$ be the Cartan involution of \&s with associated Cartan decomposition $\mathbb{S}=\mathfrak{l}$ $+\mathfrak{p}, \mathfrak{f}$ as above. Then each conjugacy class of Cartan subalgebra contains a representative which is $\theta$-stable. Thus we may restrict ourselves to considering $\theta$-stable Cartan subalgebras.

If $\mathfrak{h}$ is a $\theta$-stable Cartan subalgebra of đ্s, $\mathfrak{h}=\mathfrak{h}_{k}+\mathfrak{h}_{p}$, where $\mathfrak{h}_{k}=$ $\mathfrak{h} \cap \mathfrak{f}, \mathfrak{h}_{p}=\mathfrak{h} \cap \mathfrak{p}$. We determine the integers $c_{y}\left(w: \lambda: \mathfrak{h}^{+}\right)$by induction on $r=\operatorname{dim} \mathfrak{h}_{p}$. To perform the induction we need the following facts.

(2.1) ([2a), p. 277]). Let $\Gamma$ be a semi-regular element of noncompact type in $\mathbb{S}$. Let $\mathfrak{h}_{1}=\mathbb{S}_{\Gamma}^{+}$and $\mathfrak{h}_{2}=\mathbb{S}_{\Gamma}^{-}$be the corresponding Cartan subalgebras constructed as in [4, Volume I, p. 102], $\nu=$ $\exp \left(-\pi \sqrt{-1} / 4 \operatorname{ad}\left(X_{\Gamma}^{*}+Y_{\Gamma}^{*}\right)\right), \Phi^{+}\left(\mathbb{S}_{C}, \mathfrak{h}_{2 C}\right)=\left\{{ }^{\nu} \beta: \beta \in \Phi^{+}\left(\mathbb{S}_{C}, \mathfrak{h}_{1 C}\right)\right\}$, and $\alpha$ the unique positive real root of $\mathfrak{h}_{1}$ satisfying $\alpha(\Gamma)=0$. Let $y \in G_{c}$ satisfy $y\left(\mathfrak{t}_{c}\right)=\mathfrak{h}_{1 c}$. Let $\mathfrak{h}_{1}^{+}$and $\mathfrak{h}_{1}^{-}$be the connected components of $\mathfrak{h}_{1}^{\prime}(R)$ satisfying $\Gamma \in \operatorname{cl}\left(\mathfrak{h}_{1}^{ \pm}\right)$. Let $\mathfrak{h}_{2}^{+}$be the connected component of $\mathfrak{h}_{2}^{\prime}(R)$ containing $\Gamma$. Then $\nu y\left(\mathrm{t}_{c}\right)=\mathfrak{h}_{2 C}$, and for $w \in$ $W\left(\mathbb{S}_{c}, t_{c}\right), \lambda \in \mathscr{F}^{\prime}$,

$$
\begin{aligned}
c_{y}\left(w: \lambda: \mathfrak{h}_{1}^{+}\right)+c_{y}\left({ }^{y-1} s_{\alpha} w: \lambda: \mathfrak{h}_{1}^{+}\right) \\
\quad=c_{y}\left(w: \lambda: \mathfrak{h}_{1}^{-}\right)+c_{y}\left({ }^{y-1} s_{\alpha} w: \lambda: \mathfrak{h}_{1}^{-}\right) \\
\quad=c_{\nu y}\left(w: \lambda: \mathfrak{h}_{2}^{+}\right)+c_{\nu y}\left({ }^{y-1} s_{\alpha} w: \lambda: \mathfrak{h}_{2}^{+}\right) .
\end{aligned}
$$

(2.2) Suppose $x, y \in G_{c}$ such that $\mathfrak{h}_{c}=x\left(\mathrm{t}_{c}\right)=y\left(\mathrm{t}_{c}\right)$. Then for some

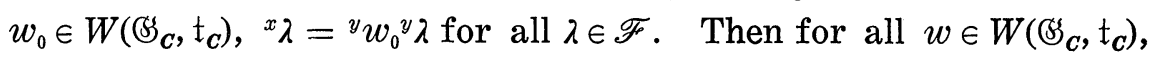
${ }^{x}(w \lambda)={ }^{y}\left(w_{0} w \lambda\right)$ and hence $c_{x}\left(w: \lambda: \mathfrak{h}^{+}\right)=\operatorname{det} w_{0} c_{y}\left(w_{0} w: \lambda: \mathfrak{h}^{+}\right)$.

(2.3) ([2a), p. 272]). For $\mathfrak{h}^{\mathfrak{h}^{+}}$, and $y$ as above, $c_{y}\left(w: \lambda: \mathfrak{h}^{+}\right)=0$ unless $\operatorname{Re}^{y}(w \lambda)(H) \leq 0$ for all $H \in \mathfrak{h}^{+}$.

(2.4) Suppose $\tilde{\mathfrak{h}}=x(\mathfrak{h})$ for some $x \in G$. Let $\tilde{\mathfrak{h}}^{+}=x\left(\mathfrak{h}^{+}\right)$and suppose $\Phi^{+}\left(\mathbb{S}_{C}, \tilde{\mathfrak{h}}_{C}\right)=\left\{{ }^{x} \alpha: \alpha \in \Phi^{+}\left(\mathscr{S}_{C}, \mathfrak{h}_{C}\right)\right\}$. Then for $H \in \mathfrak{h}^{+}$,

$$
\begin{aligned}
& T_{\lambda}(H)=T_{\lambda}\left({ }^{x} H\right)=\pi^{\mathfrak{h}}(H)^{-1} \sum_{w \in W\left(\Theta_{C},{ }^{\mathrm{t}} C^{C}\right.} \operatorname{det} w c_{y}\left(w: \lambda: \mathfrak{h}^{+}\right) \exp \left({ }^{y}(w \lambda)(H)\right) \\
& =\pi^{\tilde{\mathfrak{G}}}\left({ }^{x} H\right)^{-1} \sum_{w \in W\left({ }^{(}{ }_{C},{ }^{\mathfrak{t}} c^{)}\right.} \operatorname{det} w c_{y}\left(w: \lambda: \mathfrak{h}^{+}\right) \exp \left({ }^{x y}(w \lambda)\left({ }^{x} H\right)\right) .
\end{aligned}
$$

Therefore $c_{x y}\left(w: \lambda: \tilde{\mathfrak{h}}^{+}\right)=c_{y}\left(w: \lambda: \mathfrak{h}^{+}\right)$. 
(2.5) ([2a), p. 281]). For $s \in W\left(\otimes_{c}, t_{c}\right), t \in W(G, H)=N_{G}(H) / H, H$ the Cartan subgroup of $G$ corresponding to $\mathfrak{h}, N_{G}(H)$ the normalizer of $H$ in $G$, and $u \in W_{K}, c_{y}\left({ }^{y^{-1}} t s u^{-1}: u \lambda: t \mathfrak{h}^{+}\right)=c_{y}\left(s: \lambda: \mathfrak{h}^{+}\right)$. If we denote by $W_{R}$ the subgroup of $W\left(\mathscr{S}_{C}, \mathfrak{h}_{C}\right)$ generated by $s_{\alpha}, \alpha$ real, then $\mathfrak{h}^{\prime}(R)=\bigcup_{s \in W_{R}} s \mathfrak{h}^{+}, \mathfrak{h}^{+}$any component of $\mathfrak{h}^{\prime}(R)$. Since $W_{R} \subseteq$ $W(G, H), c_{y}\left(w: \lambda: s \mathfrak{h}^{+}\right)=c_{y}\left(y^{-1}\left(s^{-1}\right) w: \lambda: \mathfrak{h}^{+}\right)$. Thus in each case, it suffices to compute the constants for one component of $\mathfrak{h}^{\prime}(R)$.

Suppose $\operatorname{dim} \mathfrak{h}_{p}=0$. Then $\mathfrak{h}=\mathfrak{h}_{k}$ is a Cartan subalgebra of $\mathfrak{i}$ and is conjugate to $\mathfrak{t}$ by an element of $K$, say $\mathfrak{h}=k(t) . \quad \mathfrak{h}^{\prime}(R)=\mathfrak{h}$ has exactly one connected component since $\mathfrak{h}$ has no real roots. By (2.4) and (1.1)

$$
c_{k}(w: \lambda: \mathfrak{h})=c_{1}(w: \lambda: \mathfrak{t})=\left\{\begin{array}{ll}
1, & w \in W_{K} \\
0, & w \notin W_{K}
\end{array} .\right.
$$

If $y$ is any element of $G_{c}$ such that $y\left(t_{c}\right)=\mathfrak{h}_{c}$, then, choosing $w_{0}$ as in (2.3),

$$
c_{y}(w: \lambda: \mathfrak{h})=\operatorname{det} w_{0} c_{k}\left(w_{0} w: \lambda: \mathfrak{h}\right)=\left\{\begin{array}{cl}
\operatorname{det} w_{0}, & w \in w_{0}^{-1} W_{K} \\
0, & w \notin w_{0}^{-1} W_{K}
\end{array} .\right.
$$

We may now assume inductively that there is an $r \geq 1$ such that for any $\theta$-stable Cartan subalgebra $\mathfrak{h}$ with $\operatorname{dim} \mathfrak{h}_{p}<r$ we know the value of $c_{y}\left(w: \lambda: \mathfrak{h}^{+}\right)$for every component $\mathfrak{h}^{+}$of $\mathfrak{h}^{\prime}(R)$, and every $w \in W\left(\mathbb{S}_{c}, \mathfrak{t}_{c}\right)$, $\lambda \in \mathscr{F}^{\prime}$, and $y \in G_{c}$ with $\mathfrak{h}_{c}=y\left(\mathrm{t}_{c}\right)$.

Let $\dot{\mathrm{i}}=\dot{\mathrm{j}}_{k}+\dot{\mathrm{j}}_{p}$ be a $\theta$-stable Cartan subalgebra of \&s with $\operatorname{dim} \dot{\mathrm{j}}_{p}=r$, $\dot{\mathrm{i}}_{c}=y\left(\mathrm{t}_{c}\right)$, some $y \in G_{c}$. Let $\Phi_{R}$ be the set of those $\alpha \in \Phi\left(\otimes_{c}, \dot{\mathrm{I}}_{c}\right)$ which assume real values on $\dot{j}$. Then $\operatorname{dim} \Phi_{R}=\operatorname{dim} \dot{\mathrm{i}}_{p}$. Let $\dot{\mathrm{j}}^{+}$be a connected component of $\mathrm{j}^{\prime}(R)$. Define $\Phi_{R}^{+}$by $\alpha \in \Phi_{R}^{+}$if $\alpha(H)>0$ for all $H \in \dot{j}^{+}$. Then $\Phi_{R}^{+}$is a set of positive roots for $\Phi_{R} . \quad \dot{1}^{-}=\left\{-H: H \in \dot{\mathfrak{t}}^{+}\right\}$is also a component of $\mathfrak{j}^{\prime}(R)$.

Let $\left\{\beta_{1}, \cdots, \beta_{r}\right\}$ be a strongly orthogonal system of positive real roots in $\Phi_{R}^{+}$. That is, for $1 \leq i \neq j \leq r, \beta_{i} \pm \beta_{j} \notin \Phi_{R}$. Such a system of $r$ elements exists because of the correspondence between these systems and conjugacy classes of Cartan subalgebras in (8), and the fact that (5) has a compact Cartan subalgebra. (See [4, Volume I, Section 1.3.1]). The $\beta_{i}, 1 \leq i \leq r$, are in particular, mutually orthogonal, and the $H_{\beta_{i}}, 1 \leq i$ $\leq r$, form a basis for $\dot{f}_{p}$, where for any root $\alpha, H_{\alpha}$ will always denote the element of $\dot{\mathrm{I}}_{\boldsymbol{c}}$ satisfying $B\left(H_{\alpha}, H\right)=\alpha(H)$ for all $H \in \dot{\mathrm{I}}_{c}, B$ the Cartan Killing form. We also write $H_{\alpha}^{*}=2 H_{\alpha} /\langle\alpha, \alpha\rangle$. 
Set $w^{Q}=s_{\beta_{1}} \cdots s_{\beta r}$. Then $w^{Q} \dot{j}^{+}=\dot{j}^{-}$. Note that if $\lambda \in \mathscr{F}, H \in \dot{1}^{+}$, $H=H_{k}+\sum_{j=1}^{r} r_{j} H_{\beta_{j}}$ where $H_{k} \in \dot{\mathrm{I}}_{k}, r_{j} \in \boldsymbol{R}, 1 \leq j \leq r$, then $\operatorname{Re}\left\{{ }^{y} \lambda\left(w^{Q} H\right)\right\}$ $=\operatorname{Re}\left\{{ }^{y} \lambda\left(H_{k}-\sum r_{j} H_{\beta_{j}}\right)\right\}={ }^{y} \lambda\left(-\sum r_{j} H_{\beta_{j}}\right)=-\operatorname{Re}\left\{{ }^{y} \lambda(H)\right\}$. Decompose $w^{Q}$ into a product of reflections corresponding to simple roots, $w^{Q}=s_{m} \cdots s_{1}$, $s_{j}=s_{\alpha_{j}}\left\{\alpha_{1}, \cdots, \alpha_{r}\right\}$ a set of simple roots for $\Phi_{R}^{+}, 1 \leq i_{j} \leq r$ for all $1 \leq$ $j \leq m$.

For each $\alpha_{\ell}, 1 \leq \ell \leq r$, we construct a Cartan subalgebra $\dot{j}_{\ell}$ of \&s as follows. Let $\Gamma_{\ell} \in \operatorname{cl}\left(\mathfrak{l}^{+}\right)$be such that $\pm \alpha_{\ell}\left(\Gamma_{\ell}\right)=0, \alpha\left(\Gamma_{\ell}\right) \neq 0$ for any other $\alpha \in \Phi\left(\mathscr{\mho}_{c}, \dot{\pi}_{c}\right)$. Then $\Gamma_{\ell}$ is a semi-regular element of noncompact type. Let $\mathbb{S}_{\ell}$ be the centralizer of $\Gamma_{\ell}$ in $\mathbb{S}^{S}$. Then $\mathbb{S}_{\ell}=\mathfrak{c}_{\ell}+\mathfrak{l}_{\ell}$ where $\mathfrak{c}_{\ell}$ is the center of $\mathscr{G}_{\ell}$ and $\mathfrak{r}_{\ell}=\left[\mathscr{G}_{\ell}, \mathscr{G}_{\ell}\right]$ is semisimple and isomorphic to $\operatorname{sl}(2, \boldsymbol{R})$. Denote by $H_{b}^{*}, X_{b}^{*}, Y_{\ell}^{*}$ the standard basis of $\Upsilon_{\ell}$ satisfying $\left[H_{b}^{*}, X_{b}^{*}\right]=2 X_{\ell}^{*}$, $\left[H_{\ell}^{*}, Y_{\ell}^{*}\right]=-2 Y_{\ell}^{*},\left[X_{\ell}^{*}, Y_{\ell}^{*}\right]=H_{\ell}^{*}$. Then $\mathbb{S}_{\ell}^{+}=\mathfrak{c}_{\ell}+R H_{\ell}^{*}=\dot{\mathfrak{l}}$, and $\mathbb{S}_{\ell}^{-}=$ $\mathfrak{c}_{\ell}+\boldsymbol{R}\left(X_{\ell}^{*}-Y_{\ell}^{*}\right)$ is a $\theta$-stable Cartan subalgebra of $\mathbb{S}$ which we call $\dot{\mathrm{j}}_{\ell}$. $\operatorname{dim}\left(\dot{j}_{\ell} \cap \mathfrak{p}\right)=r-1$. Let $\dot{\mathfrak{l}}_{e}^{+}$be the connected component of $\dot{j}_{\ell}^{\prime}(R)$ containing $\Gamma_{\ell}$, and let $\nu_{\ell}=\exp \left(-\pi \sqrt{-1} / 4\right.$ ad $\left.\left(X_{\ell}^{*}+Y_{\ell}^{*}\right)\right)$. Then $\nu_{\ell}\left(\dot{\mathrm{i}}_{c}\right)=\dot{j}_{\ell c}$.

By (2.1), for all $w \in W\left(\mathbb{S}_{c}, t_{c}\right)$ and $\lambda \in \mathscr{F}^{\prime}$,

$$
c_{y}\left(w: \lambda: \dot{\mathrm{j}}^{+}\right)+c_{y}\left(y^{-1}\left(s_{\alpha_{\ell}}\right) w: \lambda: \mathrm{j}^{+}\right)=c_{\nu \ell y}\left(w: \lambda: \mathrm{j}_{\ell}^{+}\right)+c_{\nu \ell y}\left(y^{-1}\left(s_{\alpha_{\ell}}\right) w: \lambda: \mathrm{j}_{\ell}^{+}\right)
$$

where the terms on the right side are known by the induction hypothesis.

Let $w \in W\left(\mathbb{S}_{c}, t_{c}\right), \lambda \in \mathscr{F}^{\prime}$. Suppose there is $H \in \dot{j}^{+}$such that $\operatorname{Re}\left\{{ }^{y}(w \lambda)(H)\right\}>0 . \quad$ By $(2.2), \quad c_{y}\left(w: \lambda: \dot{\jmath}^{+}\right)=0$. Thus we may assume $\operatorname{Re}\left\{{ }^{y}(w \lambda)(H)\right\} \leq 0$ for all $H \in \dot{i}^{+}$. Since $\lambda$ is regular, there is $H_{0} \in \dot{j}^{+}$such that $\operatorname{Re}\left\{{ }^{y}(w \lambda)\left(H_{0}\right)\right\}<0$. Then $\operatorname{Re}\left\{{ }^{y}\left(y^{y-1} w^{Q} w \lambda\right)\left(H_{0}\right)\right\}=\operatorname{Re}\left\{{ }^{y}(w \lambda)\left(w^{Q} H_{0}\right)\right\}=$ $-\operatorname{Re}\left\{{ }^{y}(w \lambda)\left(H_{0}\right)\right\}>0$. Thus $c_{y}\left(y^{y-1} w^{Q} w: \lambda: \mathrm{j}^{+}\right)=0$. By using (2.1) repeatedly, we obtain

$$
\begin{aligned}
& c_{y}\left(w: \lambda: \dot{\mathrm{j}}^{+}\right)=c_{\nu i_{1} y}\left(w: \lambda: \dot{\mathrm{\dagger}}_{i_{1}}^{+}\right)+c_{\nu i_{1} y}\left(y^{-1} s_{1} w: \lambda: \dot{\mathrm{i}}_{i_{1}}^{+}\right) \\
& -c_{\nu_{i_{2}} y}\left(y^{-1} s_{1} w: \lambda: \dot{\mathrm{j}}_{i_{2}}^{+}\right)-c_{\nu_{i_{2}} y}\left(y^{-1}\left(s_{2} s_{1}\right) w: \lambda: \dot{\mathrm{j}}_{i_{2}}^{+}\right) \\
& +(-1)^{j+1}\left\{c_{v i j y} y y^{(y-1}\left(s_{j-1} \cdots s_{1}\right) w: \lambda: \dot{\mathrm{i}}_{i_{j}}^{+}\right) \\
& \left.+c_{\nu i, y}\left(y^{-1}\left(s_{j} \cdots s_{1}\right) w: \lambda: \dot{\mathfrak{j}}_{i_{j}}^{+}\right)\right\} \\
& +(-1)^{m+1}\left\{c_{v_{t_{m}} y}\left(y-1\left(s_{m-1} \cdots s_{1}\right) w: \lambda: \dot{\mathfrak{i}}_{i_{m}}^{+}\right)\right. \\
& +c_{\nu i_{m} y}\left(y^{-1} w^{Q} w: \lambda: \dot{\dagger}_{i_{m}}^{+}\right\} \text {. }
\end{aligned}
$$




\section{§3. The Constants for Special Cases}

We will now illustrate the method outlined in $\S 2$ by computing the integers $c_{y}\left(w: \lambda: \mathfrak{h}^{+}\right)$for the cases $\operatorname{dim} \mathfrak{h}_{p}=1,2$, using the notation of $\S 2$.

$\operatorname{dim} \mathfrak{h}_{p}=1$ : Suppose $\mathfrak{h}=\mathfrak{h}_{k}+\mathfrak{h}_{p}$ is a $\theta$-stable Cartan subalgebra with $\operatorname{dim} \mathfrak{h}_{p}=1$. Let $\mathfrak{h}^{+}$be a connected component of $\mathfrak{h}^{\prime}(R)$. Then $\Phi_{R}^{+}$has exactly one element, call it $\alpha$, and $\mathfrak{h}^{+}=\left\{H_{k}+r H_{\alpha}^{*}: H_{k} \in \mathfrak{h}_{k}, r \in R, r>0\right\}$. $\mathfrak{h}^{-}=\left\{H_{k}+r H_{\alpha}^{*}: H_{k} \in \mathfrak{h}_{k}, \quad r \in R, \quad r<0\right\}$ is the only other component of $\mathfrak{h}^{\prime}(R)$, and $w^{Q}=s_{\alpha}$ satisfies $s_{\alpha} \mathfrak{h}^{+}=\mathfrak{h}^{-}$. For $\Gamma$ a semi-regular element of $\mathfrak{h}$ corresponding to $\alpha$, we have $\mathfrak{h}=\mathfrak{h}_{\Gamma}^{+}$, and $\mathfrak{h}_{\Gamma}^{-} \subseteq \mathfrak{f}$ is a compact Cartan

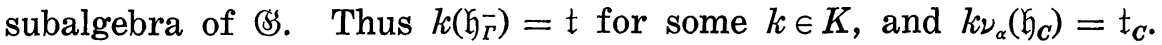

Suppose $w \in W\left(\mathscr{S}_{C}, \mathrm{t}_{C}\right), \lambda \in \mathscr{F}^{\prime}$ such that $\operatorname{Re}\left\{{ }^{\nu_{\alpha}-{ }^{-1} k^{-1}}(w \lambda)(H)\right\} \leq 0$ for all $H \in \mathfrak{h}^{+}$. Then by (2.8), $c_{\nu_{\alpha}-1_{k-1}}\left(w: \lambda: \mathfrak{h}^{+}\right)=c_{k-1}\left(w: \lambda: \mathfrak{h}_{\Gamma}^{-}\right)+c_{k-1}\left({ }^{\left(k_{\nu \alpha}\right)} s_{\alpha} w: \lambda: \mathfrak{h}_{\bar{r}}\right)$ where by (2.6),

$$
c_{k-1}\left(w: \lambda: \mathfrak{h}_{\bar{\Gamma}}\right)= \begin{cases}1, & w \in W_{K} \\ 0, & w \notin W_{K}\end{cases}
$$

and

$$
\left.c_{k-1}{ }^{(k \nu \alpha)} s_{\alpha} w: \lambda: \mathfrak{G}_{\bar{\Gamma}}\right)= \begin{cases}1, & { }^{(k \nu \alpha)} s_{\alpha} w \in W_{K} \text { iff } w \in{ }^{(k \nu \alpha)} s_{\alpha} W_{K} \\ 0, & \text { otherwise }\end{cases}
$$

$W_{K} \neq{ }^{\left(k_{\nu \alpha}\right)} s_{\alpha} W_{K}$ as cosets of $W_{K}$ in $W\left(\mho_{C}, t_{C}\right)$ since ${ }^{\nu \alpha} \alpha$ is a singular imaginary root of $\mathfrak{h}_{\bar{\Gamma}}$ and hence ${ }^{\left(k \nu_{\alpha}\right)} \alpha$ is a singular imaginary root of $t$.

For any $H \in \mathfrak{h}^{+}, H=H_{k}+r H_{\alpha}^{*}, \operatorname{Re}\left\{{ }^{\left(k \nu_{\alpha}\right)^{-1}}(w \lambda)(H)\right\}={ }^{\left(k \nu_{\alpha}\right)-1}(w \lambda)\left(r H_{\alpha}^{*}\right)=$ $r^{\left(k \nu_{\alpha}\right)-1}(w \lambda)\left(H_{\alpha}^{*}\right)$. Thus we have:

$$
c_{\left(k \nu_{\alpha}\right)-1}\left(w: \lambda: \mathfrak{h}^{+}\right)= \begin{cases}1, & \left({ }^{\left(k \nu_{\alpha}\right)-1}(w \lambda)\left(H_{\alpha}^{*}\right)<0, w \in W_{K} \cup^{\left(k \nu_{\alpha}\right)} s_{\alpha} W_{K}\right. \\ 0, & \text { otherwise . }\end{cases}
$$

For arbitrary $y \in G_{c}, y\left(\mathfrak{t}_{c}\right)=\mathfrak{h}_{c}$, we can use (2.2) to determine $c_{y}\left(w: \lambda: \mathfrak{h}^{+}\right)$.

$\operatorname{dim} \mathfrak{h}_{p}=2$ : Suppose $\mathfrak{h}=\mathfrak{h}_{k}+\mathfrak{h}_{p}$ is a $\theta$-stable Cartan subalgebra with $\operatorname{dim} \mathfrak{h}_{p}=2$. Then $\Phi_{R}$ is a two-dimensional root system containing a pair of strongly orthogonal roots. Thus $\Phi_{R}$ is of type $A_{1} \times A_{1}, B_{2}$, or $G_{2}$. We compute the constants separately for each of these three cases. $A_{1} \times A_{1}$ : Let $\mathfrak{h}^{+}$be a connected component of $\mathfrak{h}^{\prime}(R)$. Thus $\Phi_{R}^{+}$has exactly two elements, $\alpha_{1}$ and $\alpha_{2}$, which are orthogonal and form a set of simple roots. Then $\mathfrak{h}_{p}=\left\{r_{1} H_{\alpha_{1}}^{*}+r_{2} H_{\alpha_{2}}^{*}: r_{i} \in R, i=1,2\right\}$.

$$
\mathfrak{h}^{+}=\left\{H_{k}+r_{1} H_{\alpha_{1}}^{*}+r_{2} H_{\alpha_{2}}^{*}: H_{k} \in \mathfrak{h}_{k}, r_{i} \in R, r_{i}>0, i=1,2\right\}
$$

and 


$$
\mathfrak{h}^{-}=\left\{H_{k}+r_{1} H_{\alpha_{1}}^{*}+r_{2} H_{\alpha_{2}}^{*}: H_{k} \in \mathfrak{h}_{k}, r_{i} \in \boldsymbol{R}, r_{i}<0, i=1,2\right\}=s_{\alpha_{2}} s_{\alpha_{1}} \mathfrak{h}^{+} .
$$

Thus $w^{Q}=s_{\alpha_{2}} s_{\alpha_{1}}$ is a decomposition of $w^{Q}$ into simple reflections. Let $\dot{\lambda}_{1}$ and $\dot{\lambda}_{2}$ be the Cartan subalgebras constructed as in $\S 2$.

Then $\nu_{i}\left(\mathfrak{h}_{c}\right)=\left(\dot{\mathfrak{j}}_{i}\right)_{c}, i=1,2$, and $\mathfrak{t}_{1 c}=\nu_{2} \nu_{1}\left(\mathfrak{h}_{c}\right)=\nu_{2}\left(\dot{\mathfrak{t}}_{1 c}\right)=\nu_{1} \nu_{2}\left(\mathfrak{h}_{c}\right)=\nu_{1}\left(\dot{\mathfrak{t}}_{2 c}\right)$ is a compact Cartan subalgebra of $\mathbb{B S}_{c}$. Let $k \in K$ satisfy $k\left(\mathrm{t}_{1}\right)=\mathrm{t}$. Then $\mathfrak{h}_{c}=\nu_{2}^{-1} \nu_{1}^{-1} k^{-1}\left(\mathrm{t}_{c}\right)=\nu_{1}^{-1} \nu_{2}^{-1} k^{-1}\left(\mathrm{t}_{c}\right)$. Denote $\nu_{1}^{-1} \nu_{2}^{-1} k^{-1}=\nu_{2}^{-1} \nu_{1}^{-1} k^{-1}$ by $y$.

Suppose $w \in W\left(\mathscr{S}_{c}, \mathrm{t}_{c}\right), \lambda \in \mathscr{F}^{\prime}$, so that $\operatorname{Re}\left\{{ }^{y}(w \lambda)(H)\right\} \leq 0$ for all $H \in \mathfrak{G}^{+}$. Then

$$
\begin{aligned}
c_{y}\left(w: \lambda: \mathfrak{h}^{+}\right)= & c_{\nu_{2}-1_{k}-1}\left(w: \lambda: \dot{\mathrm{j}}_{1}^{+}\right)+c_{\nu_{2}-1_{k}-1}\left(y^{-1} s_{\alpha_{1}} w: \lambda: \dot{\mathrm{j}}_{1}^{+}\right) \\
& -c_{\nu_{1}-1 k^{-1}}\left(y^{-1} s_{\alpha_{1}} w: \lambda: \dot{\mathfrak{j}}_{2}^{+}\right)-c_{\nu_{1}-k_{k}-1}\left(y^{-1}\left(s_{\alpha_{2}} s_{\alpha_{1}}\right) w: \lambda: \dot{\mathfrak{j}}_{2}^{+}\right),
\end{aligned}
$$

where by (3.1):

$$
\begin{aligned}
& c_{\nu_{2}-1 k^{-1}}\left(w: \lambda: \dot{\mathrm{j}}_{1}^{+}\right)= \begin{cases}1, & \nu_{2}^{-1 k^{-1}}(w \lambda)\left(H_{\alpha_{2}}^{*}\right)<0, w \in W_{K} \cup^{y^{-1}} s_{\alpha_{2}} W_{K} \\
0, & \text { otherwise }\end{cases}
\end{aligned}
$$

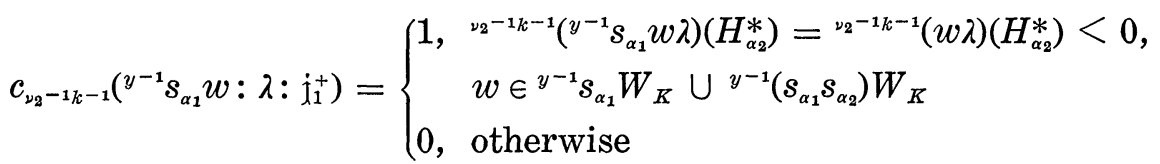

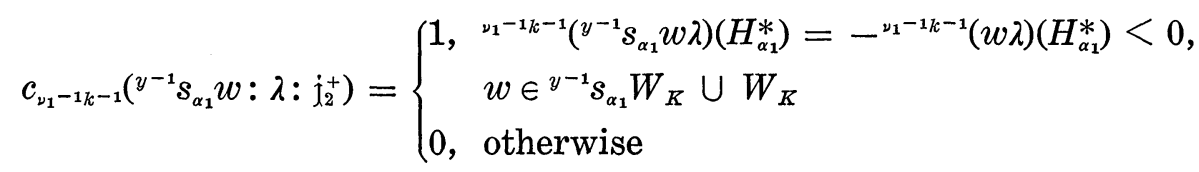

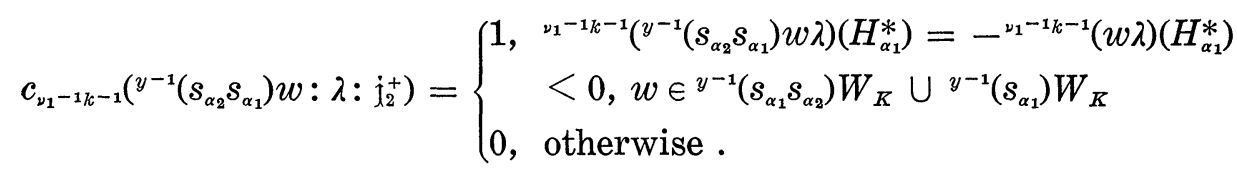

Thus, since ${ }^{\nu_{i}-1{ }^{-1}-1}(w \lambda)\left(H_{\alpha_{i}}^{*}\right)={ }^{y}(w \lambda)\left(H_{\alpha_{i}}^{*}\right), i=1,2$, and the cosets $W_{K}$, ${ }^{y-1} s_{\alpha_{1}} W_{K},{ }^{y-1} s_{\alpha_{2}} W_{K}$, and ${ }^{y-1}\left(s_{\alpha_{1}} s_{\alpha_{2}}\right) W_{K}$ are distinct,

$$
c_{y}\left(w: \lambda: \mathfrak{h}^{+}\right)= \begin{cases}1, & y(w \lambda)\left(H_{\alpha_{i}}^{*}\right)<0, i=1,2, \\ & w \in W_{K} \cup^{y^{-1}} s_{\alpha_{1}} W_{K} \cup^{y^{-1}} s_{\alpha_{2}} W_{K} \cup^{y^{-1}}\left(s_{\alpha_{1}} s_{\alpha_{2}}\right) W_{K} \\ 0, & \text { otherwise . }\end{cases}
$$

$B_{2}$ : Let $\mathfrak{h}^{+}$be a connected component of $\mathfrak{h}^{\prime}(R)$. Then $\Phi_{R}^{+}$has a set of simple roots, $\left\{\alpha_{1}, \alpha_{2}\right\}$. Assume that $\alpha_{1}$ is the long root. Then $\Phi_{R}^{+}=$ $\left\{\alpha_{1}, \alpha_{2}, \beta_{2}=\alpha_{1}+\alpha_{2}, \beta_{1}=\alpha_{1}+2 \alpha_{2}\right\}$ where $\left\langle\alpha_{i}, \beta_{i}\right\rangle=0, i=1,2 . \mathfrak{h}_{p}=\left\{r H_{\alpha_{1}}^{*}+\right.$ $\left.s H_{\beta_{1}}^{*}: r, s \in \boldsymbol{R}\right\}, \mathfrak{h}^{+}=\left\{H_{k}+r H_{\alpha_{1}}^{*}+s H_{\beta_{1}}^{*}: H_{k} \in \mathfrak{h}_{k}, s>r>0\right\}$, and $\mathfrak{h}^{-}=\left\{H_{k}+\right.$ $\left.r H_{\alpha_{1}}^{*}+s H_{\beta_{1}}^{*}: H_{k} \in \mathfrak{h}_{k}, 0>r>s\right\}=s_{\alpha_{2}} s_{\alpha_{1}} s_{\alpha_{2}} s_{\alpha_{1}} \mathfrak{h}^{+}$. Thus $w^{Q}=s_{\alpha_{2}} s_{\alpha_{1}} s_{\alpha_{2}} s_{\alpha_{1}}$. 
Let $\dot{\mathrm{I}}_{1}$ and $\dot{\mathrm{f}}_{2}$ be constructed as in $\S 2$ corresponding to $\alpha_{1}$ and $\alpha_{2}$ respectively. Then ${ }^{\nu} \beta_{i}$ is the unique positive real root of $\dot{i}_{i}$, and using a semi-regular element $\Gamma_{i}$ of $\dot{f}_{i}$ corresponding to ${ }^{\nu} \beta_{i}$, we obtain compact Cartan subalgebras $t_{i}=\mathbb{S}_{\bar{F}_{i}}$ together with isomorphisms $\mu_{i}:\left(\dot{\mathfrak{f}}_{i}\right)_{\boldsymbol{c}} \rightarrow\left(\mathrm{t}_{i}\right)_{c}$, $i=1,2$. Pick $k_{1} \in K$ such that $k_{1}\left(t_{1}\right)=t$. Then there is $k_{2} \in K$ such that $k_{2}\left(t_{2}\right)=t$ and $k_{1} \mu_{1} \nu_{1}=k_{2} \mu_{2} \nu_{2}$ as isomorphisms from $\mathfrak{h}_{\boldsymbol{c}}$ to $\mathrm{t}_{\boldsymbol{c}}$. Denote this isomorphism by $y$.

Fix $w \in W\left(\mathbb{S}_{c}, \mathrm{t}_{c}\right), \lambda \in \mathscr{F}^{\prime}$. Let $n={ }^{y-1}(w \lambda)\left(H_{\alpha_{1}}^{*}\right), m={ }^{y-1}(w \lambda)\left(H_{\beta_{1}}^{*}\right)$. Then $\operatorname{Re}\left\{y^{-1}(w \lambda)\left(H_{k}+r H_{\alpha_{1}}^{*}+s H_{\beta_{1}}^{*}\right)\right\}=r n+s m$. If $m>0$ or $n>-m$, there is $H \in \mathfrak{h}^{+}$such that $\operatorname{Re}\left\{y^{-1}(w \lambda)(H)\right\}>0$. Assume this is not the case. Then

$$
\begin{aligned}
& c_{y^{-1}}\left(w: \lambda: \mathfrak{h}^{+}\right)=c_{\nu_{1} y^{-1}}\left(w: \lambda: \dot{\jmath}_{1}^{+}\right)+c_{\nu_{1} y^{-1}}\left({ }^{y} s_{\alpha_{1}} w: \lambda: \dot{\jmath}_{1}^{+}\right)-c_{\nu_{2} y^{-1}}\left({ }^{y} s_{\alpha_{1}} w: \lambda: \dot{\jmath}_{2}^{+}\right) \\
& -c_{\nu_{2} y^{-1}}\left({ }^{y}\left(s_{\alpha_{2}} s_{\alpha_{1}}\right) w: \lambda: \dot{\mathrm{j}}_{2}^{+}\right)+c_{\nu_{1} y^{-1}}\left({ }^{y}\left(s_{\alpha_{2}} s_{\alpha_{1}}\right) w: \lambda: \dot{\mathrm{j}}_{1}^{+}\right) \\
& +c_{\nu_{1} y^{-1}}\left({ }^{y}\left(s_{\alpha_{1}} s_{\alpha_{2}} s_{\alpha_{1}}\right) w: \lambda: \dot{\mathrm{l}}_{1}^{+}\right)-c_{\nu_{2} y^{-1}}\left({ }^{y}\left(s_{\alpha_{1}} s_{\alpha_{2}} s_{\alpha_{1}}\right) w: \lambda: \dot{\mathrm{l}}_{2}^{+}\right) \\
& -c_{\nu 2 y-1}\left({ }^{y} w^{Q} w: \lambda: \dot{j}_{2}^{+}\right) \text {. }
\end{aligned}
$$

The constants for $\dot{i}_{1}^{+}$and $\dot{i}_{2}^{+}$can be evaluated using the facts that $\nu_{i} y^{-1}=\left(k_{i} \mu_{i}\right)^{-1}$ as isomorphisms from $t_{c}$ to $\left(\dot{\mathfrak{f}}_{i}\right)_{c}$, and that the cosets $W_{K}$, ${ }^{y} S_{\alpha_{1}} W_{K},{ }^{y} S_{\beta_{1}} W_{K},{ }^{y}{S_{\beta_{2}}}_{W_{K}}={ }^{y}\left(s_{\alpha_{1}} s_{\beta_{1}}\right) W_{K}$ are distinct, but that ${ }^{y} s_{\alpha_{2}} W_{K}=W_{K}$ since ${ }^{y} \alpha_{2}$ is a compact root of $t$. We obtain the following table of values for $c_{y-1}\left(w: \lambda: \mathfrak{h}^{+}\right)$where $n$ and $m$ are as defined above.

$\begin{array}{lrc} & w \in W_{K} \cup{ }^{y} s_{\beta_{2}} W_{K} & w \in{ }^{y} s_{\alpha_{1}} W_{K} \cup{ }^{y} s_{\beta_{1}} W_{K} \\ 0>m>n & 1 & 1 \\ 0>n>m & 1 & -1 \\ 0<n<-m & 2 & 0 \\ m>0 \text { or } n>-m & 0 & 0\end{array}$

$G_{2}$ : Let $\mathfrak{h}^{+}$be a connected component of $\mathfrak{h}^{\prime}(R)$. Let $\left\{\alpha_{1}, \alpha_{2}\right\}$ be the set of simple roots for $\Phi_{R}^{+}, \alpha_{2}$ the long root. Then $\Phi_{R}^{+}=\left\{\alpha_{1}, \alpha_{2}, \alpha_{3}=\alpha_{1}+\alpha_{2}\right.$, $\left.\beta_{2}=2 \alpha_{1}+\alpha_{2}, \beta_{3}=3 \alpha_{1}+\alpha_{2}, \beta_{1}=3 \alpha_{1}+2 \alpha_{2}\right\}$, where $\left\langle\alpha_{i}, \beta_{i}\right\rangle=0, i=1,2,3$. $\mathfrak{h}_{p}=\left\{r H_{\alpha_{1}}^{*}+s H_{\beta_{1}}^{*}: r, s \in \boldsymbol{R}\right\}, \mathfrak{h}^{+}=\left\{H_{k}+r H_{\alpha_{1}}^{*}+s H_{\beta_{1}}^{*}: H_{k} \in \mathfrak{h}_{k}, \quad s>3 r>0\right\}$ and $\mathfrak{h}^{-}=s_{\alpha_{1}} s_{\alpha_{2}} s_{\alpha_{1}} s_{\alpha_{2}} s_{\alpha_{1}} s_{\alpha_{2}} \mathfrak{G}^{+}$. Thus $w^{Q}=\left(s_{\alpha_{1}} s_{\alpha_{2}}\right)^{3}$. Let $\dot{\mathfrak{l}}_{1}$ and $\dot{\mathfrak{f}}_{2}$ be constructed as in $\S 2$ and define $t_{i}, \mu_{i}$ as in the $B_{2}$ case for $i=1,2$. Let $y=k_{1} \mu_{1} \nu_{1}$ where $k_{1} \in K$ satisfies $k_{1}\left(t_{1}\right)=t$.

Fix $w \in W\left(\mho_{c}, \ddagger_{c}\right), \quad \lambda \in \mathscr{F}^{\prime}$. Let $n=y^{-1}(w \lambda)\left(H_{\alpha_{1}}^{*}\right), \quad m=y^{-1}(w \lambda)\left(H_{\beta_{1}}^{*}\right)$. Then $\operatorname{Re}\left\{y^{y-1}(w \lambda)\left(H_{k}+r H_{\alpha_{1}}^{*}+s H_{\beta_{1}}^{*}\right)\right\}=r n+s m$. If $m>0$ or $n>-3 m$, there is $H \in \mathfrak{h}^{+}$such that $\operatorname{Re}\left\{{ }^{y-1}(w \lambda)(H)\right\}>0$. Otherwise, $c_{y^{-1}}\left(w: \lambda: \mathfrak{h}^{+}\right)$ 
is given as a sum of twelve terms from (2.8). Since $\nu_{1} y^{-1}=\left(k_{1} \mu_{1}\right)^{-1}$, $c_{\nu_{1} y^{-1}}\left(w^{\prime}: \lambda:{\dot{\lambda_{1}}}_{1}^{+}\right)$is given directly by (3.1) for any $w^{\prime} \in W\left(\mathbb{S}_{c}, \mathrm{t}_{c}\right)$.

However, there is no $k_{2} \in K$ with $k_{2}\left(t_{2}\right)=t$ and $\nu_{2} y^{-1}=\left(k_{2} \mu_{2}\right)^{-1}$. However, there is $k_{2} \in K$ such that $k_{2}\left(\mathrm{t}_{2}\right)=\mathrm{t}$ and ${ }^{\left(v_{2} y-1\right)} \lambda={ }^{\left(k_{2} \mu_{2}\right)-1}\left(v\left(s_{\beta_{1}} s_{\alpha_{3}}\right) \lambda\right)$ for all $\lambda \in \mathscr{F}^{\prime}$. Thus, using (2.2) together with (3.1) we obtain

$c_{\nu_{2} y-1}\left(w^{\prime}: \lambda: j_{2}^{+}\right)=\left\{\begin{array}{cl}\operatorname{det}^{y}\left(s_{\beta_{1}} s_{\alpha_{3}}\right)=1, & { }^{\nu_{2} y^{-1}}(w \lambda)\left(H_{\beta_{2}}^{*}\right)<0, w \in{ }^{y} S_{\alpha_{1}} W_{K} \cup{ }^{y} S_{\alpha_{3}} W_{K} \\ 0, & \text { otherwise }\end{array}\right.$

The cosets $W_{K},{ }^{y} s_{\alpha_{1}} W_{K}={ }^{y} s_{\beta_{1}} W_{K}$, and ${ }^{y} s_{\alpha_{3}} W_{K}={ }^{y} s_{\beta_{3}} W_{K}$ are distinct.

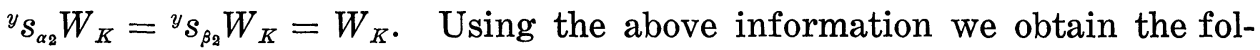
lowing table for the values of $c_{y-1}\left(w: \lambda: \mathfrak{h}^{+}\right)$.

$\begin{array}{lrcc} & w \in W_{K} & w \in{ }^{y} \mathcal{S}_{\alpha_{1}} W_{K} & w \in{ }^{y} s_{\alpha_{3}} W_{K} \\ 0>3 m>n & 2 & 2 & 0 \\ 0>m>n>3 m & 0 & 2 & -2 \\ m<n<0 & 0 & 4 & 0 \\ 0<n<-m & -2 & 2 & 0 \\ 0<-m<n<-3 m & 0 & 2 & 2 \\ m>0 \text { or } n>-3 m & 0 & 0 & 0\end{array}$

In general, the constants $c_{y}\left(w: \lambda: \mathfrak{h}^{+}\right)$become increasingly complicated as $\operatorname{dim} \mathfrak{h}_{p}$ increases. However, in the case that (f) has exactly one conjugacy class of Cartan subalgebra corresponding to each possible dimension for $\mathfrak{h}_{p}$, the constants can be computed completely. There are three infinite families of simple real Lie algebras which have this property, including $s u(p, q), p \geq q \geq 1$. The others are of types CII and DIII. ([4, Volume I, Section 1.3.1]).

Thus suppose $\mathbb{S}=\mathfrak{f}+\mathfrak{p}$ is a simple real Lie algebra with split rank $n, \mathfrak{A}$ a Cartan subalgebra of $\mathbb{6}$ with $\mathfrak{A}_{p}=\mathfrak{A} \cap \mathfrak{p}$ of dimension $n$. Assume the set $\Phi_{R}$ of real roots of $\left(\mathscr{S}_{c}, \mathfrak{A}_{c}\right)$ is of type $A_{1} \times \cdots \times A_{1}, n$ copies. Denote the real roots by $\left\{\alpha_{1}, \cdots, \alpha_{n}\right\}$. They are mutually orthogonal and form a set of simple roots for the root system $\Phi_{R}$. We can write $\mathfrak{Q}=$ $\mathfrak{A}_{k}+\sum_{i=1}^{n} \boldsymbol{R} \boldsymbol{H}_{\alpha_{i}}^{*}, \mathfrak{A}_{k}=\mathfrak{A} \cap \mathfrak{f}$. Representatives of each conjugacy class of Cartan subalgebras of $\mathbb{S}$ are $\mathfrak{X}=\mathfrak{h}_{0}, \mathfrak{h}_{1}, \cdots, \mathfrak{h}_{n}=t$, where $\mathfrak{h}_{\ell}=\mathfrak{A}_{k}+$ $\sum_{i=1}^{\ell} \boldsymbol{R}\left(X_{\alpha_{i}}^{*}-Y_{\alpha_{i}}^{*}\right)+\sum_{i=\ell+1}^{n} R H_{\alpha_{i}}^{*}$. (Here $X_{\alpha_{i}}^{*}$ and $Y_{\alpha_{i}}^{*}$ denote the elements of the root spaces $\mathscr{B S}^{\alpha_{i}}$ and $\mathbb{S}^{-\alpha_{i}}$ respectively which satisfy $\left[H_{\alpha_{i}}^{*}, X_{\alpha_{i}}^{*}\right]=$ $2 X_{\alpha_{i}}^{*} ;\left[H_{\alpha_{i}}^{*}, Y_{\alpha_{i}}^{*}\right]=-2 Y_{\alpha_{i}}^{*} ;\left[X_{\alpha_{i}}^{*}, Y_{\alpha_{i}}^{*}\right]=H_{\alpha_{i}}^{*}$. Let 


$$
\nu_{i}=\exp \left(-\pi \sqrt{-1} / 4 \mathrm{ad}\left(X_{\alpha_{i}}^{*}+Y_{\alpha_{i}}^{*}\right)\right), \quad \mu_{\ell}=\nu_{n} \cdots \nu_{\ell+1} .
$$

Then $\mu_{\ell}\left(\mathfrak{h}_{\ell}\right)_{c}=\mathfrak{t}_{c}$. Let $\mathfrak{h}_{\ell}^{+}=\left\{H_{k}+\sum_{i=\ell+1}^{n} r_{i} H_{\alpha_{i}}^{*}: H_{k} \in \mathfrak{h}_{\ell} \cap \mathfrak{l}, r_{i} \in \boldsymbol{R}, r_{i}>0\right.$ for $i=\ell+1, \cdots, n\}$.

We assume inductively that for $j>\ell, \lambda \in \mathscr{F}^{\prime}, w \in W\left(\mathbb{S}_{c}, t_{c}\right)$,

$$
c_{\mu_{j}-1}\left(w: \lambda: \mathfrak{h}_{j}^{+}\right)=\left\{\begin{array}{l}
1,{ }^{\mu_{j}^{-1}}(w \lambda)\left(H_{\alpha_{i}}^{*}\right)<0, i=j+1, \cdots, n, \\
\quad w \in\left\langle s_{\alpha_{j+1}}, \cdots, s_{\alpha_{n}}\right\rangle W_{K} \\
0, \text { otherwise }
\end{array}\right.
$$

where $\left\langle s_{\alpha_{j+1}}, \cdots, s_{\alpha_{n}}\right\rangle W_{K}$ denotes the subgroup of $W\left(\mathbb{S}_{c}, \mathrm{t}_{c}\right)$ generated by $W_{K}$ together with the reflections ${ }^{\mu_{0}}\left(s_{\alpha_{i}}\right), i=j+1, \cdots, n$. We have already proved this for the cases $j=n, n-1, n-2$ in (2.6), (3.1), and (3.2) respectively.

We know $c_{\mu_{\ell}^{-1}}\left(w: \lambda: \mathfrak{h}_{\ell}^{+}\right)=0$ if $\operatorname{Re}\left\{{ }^{\mu} \bar{\ell}^{-1}(w \lambda)(H)\right\}>0$ for any $H \in \mathfrak{h}_{\ell}^{+}$. Write $H=H_{k}+\sum_{i=\ell+1}^{n} r_{i} H_{\alpha i}^{*}, H_{k} \in \mathfrak{h}_{\ell} \cap \mathfrak{f}, r_{i}>0, i=\ell+1, \cdots, n$. Then

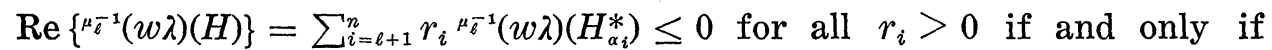
${ }^{\mu} \bar{\ell}^{-1}(w \lambda)\left(H_{\alpha_{i}}^{*}\right)<0$ for all $i$. (By the regularity of $\lambda,{ }^{\mu} \bar{\ell}^{-1}(w \lambda)\left(H_{\alpha_{i}}^{*}\right) \neq 0$ for

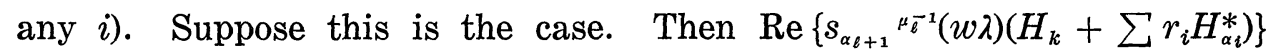
$=-r_{\ell+1}{ }^{\mu_{\bar{\ell}}^{-1}}(w \lambda)\left(H_{\alpha_{\ell+1}}^{*}\right)+\sum_{i=\ell+2}^{n} r_{i}{ }^{\mu-1}(w \lambda)\left(H_{\alpha_{i}}^{*}\right)$, and there are $r_{i}>0, i=\ell$ $+1, \cdots, n$ for which this is strictly positive, since by assumption, $\mu_{\ell}^{-1}(w \lambda)\left(H_{\alpha_{\ell+1}}^{*}\right)<0$.

Thus $c_{\mu_{\ell}-1}\left({ }^{\mu_{\ell}}\left(s_{\alpha_{\ell+1}}\right) w: \lambda: \mathfrak{h}_{\ell}^{+}\right)=0$, and so

$$
\begin{aligned}
c_{\mu_{\ell}^{-1}}\left(w: \lambda: \mathfrak{h}_{\ell}^{+}\right)= & c_{\mu_{\bar{\ell}+1}^{-1}}\left(w: \lambda: \mathfrak{h}_{\ell+1}^{+}\right)+c_{\mu_{\ell+1}^{-1}}\left({ }^{\mu_{\ell}}\left(s_{\alpha_{\ell+1}}\right) w: \lambda: \mathfrak{h}_{\ell+1}^{+}\right) \\
& = \begin{cases}1, & { }^{\mu-1}(w \lambda)\left(H_{\alpha_{j}}^{*}\right)<0, j=\ell+2, \cdots, n, \\
& w \in\left\langle s_{\alpha_{\ell+2}}, \cdots, s_{\alpha_{n}}\right\rangle W_{K} \cup^{\mu_{\ell+1}} s_{\alpha_{\ell+1}}\left\langle s_{\alpha_{\ell+2}}, \cdots, s_{\alpha_{n}}\right\rangle W_{K} \\
0, & \text { otherwise } .\end{cases}
\end{aligned}
$$

Thus we have, by induction, for any $\ell=0, \cdots, n$,

$$
c_{\mu_{\ell}^{-1}}\left(w: \lambda: \mathfrak{h}_{\ell}^{+}\right)= \begin{cases}1, & { }^{\mu_{\ell}^{-1}}(w \lambda)\left(H_{\alpha_{\jmath}}^{*}\right)<0, j=\ell+1, \cdots, n, \\ & w \in\left\langle s_{\alpha_{\ell+1}}, \cdots, s_{\alpha_{n}}\right\rangle W_{K} \\ 0, & \text { otherwise. }\end{cases}
$$

\section{§4. The Constants on $G$}

We use the notation of $\S 1$. Let $\tau \in L_{T}$. Denote the corresponding character of $T$ by $\xi_{\tau}$. Thus, for $H \in t, \xi_{\tau}(\exp H)=\exp (\tau(H))$. For any Cartan subgroup $H$ of $G$, let $\Delta_{H}$ be defined by 


$$
\Delta_{H}(h)=\xi_{\rho}(h) \prod_{\alpha \in \Phi+\left(\Theta_{C}, \mathfrak{G}_{C}\right)}\left(1-\xi_{\alpha}\left(h^{-1}\right)\right), \quad h \in H
$$

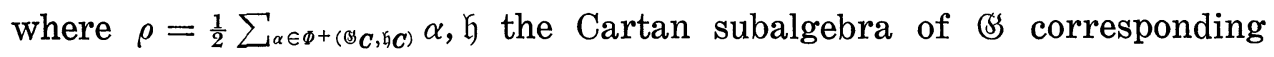
to $H$.

Then for $t \in T^{\prime}$,

$$
\Theta_{\tau}(t)=\Delta_{T}(t)^{-1} \sum_{w \in W_{K}} \operatorname{det} w \xi_{w \tau}(t)
$$

To compute the expression for $\Theta_{\tau}$ on other Cartan subgroups, we use the method and notation of [2a), § 23].

Let $H$ be a $\theta$-stable Cartan subgroup of $G$ with Cartan subalgebra $\mathfrak{h}=\mathfrak{h}_{k}+\mathfrak{h}_{p}, \operatorname{dim} \mathfrak{h}_{p}=1, H^{*}$ a connected component of $H^{\prime}(R) . \quad H^{*}=H_{I}^{*} H_{R}^{*}$, $H_{I}^{*}$ a connected component of $H_{I}=H \cap K, H_{R}^{*} \subseteq H_{R}=\exp \left(\mathfrak{h}_{p}\right)$. Note $H_{I}=Z\left(H_{R}\right) H_{I}^{0}, H_{I}^{0}$ the connected component of the identity, $Z\left(H_{R}\right)=$ $\left\{I, \gamma_{\alpha}\right\}, \alpha$ the unique positive real root of $\left(\otimes_{C}, \mathfrak{h}_{C}\right)$. (For any real root $\alpha$, $\left.\gamma_{\alpha}=\exp \left(\pi \sqrt{-1} H_{\alpha}^{*}\right)=\exp \left(\pi\left(X_{\alpha}^{*}-Y_{\alpha}^{*}\right)\right) \in \exp \left(\sqrt{-1} \mathfrak{h}_{p}\right) \cap K\right) . \quad H_{I}$ is connected if and only if $\gamma_{\alpha} \in H_{I}^{0}=\exp \left(\mathfrak{h}_{k}\right)$.

We will assume $H_{I}^{0} \subseteq T$. Let 3 denote the centralizer of $H_{I}^{*}$ in (s) where $H_{I}^{*}=H_{I}^{0}$ or $\gamma_{\alpha} H_{I}^{0}$. In either case, $3=\mathfrak{h}+()^{\alpha}+\mathbb{S}^{-\alpha}$, where for any root $\alpha, \mathbb{B S}^{\alpha}=\mathbb{B} \cap \cap \mathbb{B S}_{C}^{\alpha}, \mathbb{S}_{C}^{\alpha}$ the root space of $\alpha$ in $\mathbb{S}_{c}$, Then 3 is a reductive Lie algebra with Cartan subalgebras $\mathfrak{h}$ and $t$, where $\mathrm{t}_{c}=\nu_{\alpha}\left(\mathfrak{h}_{c}\right)$, $\nu_{\alpha}=\exp \left(-\pi \sqrt{-1} / 4 \operatorname{ad}\left(X_{\alpha}^{*}+Y_{\alpha}^{*}\right)\right) . \quad\left(B_{c}, \mathfrak{h}_{c}\right)$ has exactly one positive root, $\alpha$, and $W\left(3_{c}, \mathfrak{h}_{c}\right)=\left\{I, s_{\alpha}\right\} . \quad W_{K} \cap W\left(3_{c}, \mathfrak{t}_{c}\right)=\{I\}$.

Thus if $h_{1} \in H_{I}^{*}, h_{2} \in H_{R}^{*}, h_{1} h_{2} \in H^{\prime}$,

$$
\begin{aligned}
& \Delta_{H}\left(h_{1} h_{2}\right) \Theta_{\tau}\left(h_{1} h_{2}\right) \\
& \quad=\sum_{w \in W_{K}} \operatorname{det} w \xi_{w \tau}\left(h_{1}\right) \sum_{s \in\left\{I, s_{\alpha}\right\}} \operatorname{det} s c_{\tau}\left(s: w: H^{*}\right) \exp \left(s^{\nu \alpha^{-1}}(w \tau)\left(\log h_{2}\right)\right) \\
& \quad=\sum_{w \in W_{K}} \operatorname{det} w \xi_{w \tau}\left(h_{1}\right) c\left(w \tau: \mathfrak{h}^{*}\right) \exp \left(-\left.\right|^{\nu \alpha}(w \tau)\left(\log h_{2}\right) \mid\right)
\end{aligned}
$$

where $\mathfrak{h}^{*}$ is the component of $\mathfrak{h}^{\prime}(R)$ corresponding to $H^{*}$ under the exponential map. For $\mathfrak{h}^{*}=\mathfrak{h}^{+}=\left\{H_{k}+r H_{\alpha}^{*}: H_{k} \in \mathfrak{h}_{k}, r>0\right\}, \tau \in L_{T}$,

$$
c\left(\tau: \mathfrak{h}^{+}\right)=\left\{\begin{aligned}
1, & { }^{{ }^{\alpha}-1} \tau\left(H_{\alpha}^{*}\right)<0 \\
-1, & { }^{{ }^{\alpha}-1} \tau\left(H_{\alpha}^{*}\right)>0 \\
0, & { }^{\nu_{\alpha}-1} \tau\left(H_{\alpha}^{*}\right)=0 .
\end{aligned}\right.
$$

For $\mathfrak{h}^{-}=s_{\alpha} \mathfrak{h}^{+}, c\left(\tau: \mathfrak{h}^{-}\right)=-c\left(\tau: \mathfrak{h}^{+}\right)$for all $\tau \in L_{T}$.

Now suppose $H$ is a Cartan subgroup of $G$ with Cartan subalgebra $\mathfrak{h}=\mathfrak{h}_{k}+\mathfrak{h}_{p}, \operatorname{dim} \mathfrak{h}_{p}=2, H^{*}$ a connected component of $H^{\prime}(R)$. Again, 
we must consider the three cases where $\Phi_{R}$ is of type $A_{1} \times A_{1}, B_{2}$, or $G_{2}, \Phi_{R}$ the set of real roots in $\Phi\left(\mho_{c}, \mathfrak{h}_{c}\right)$. We use the notation of $\S 3$. We assume $H^{*}=H_{I}^{*} H_{R}^{*}, H_{I}^{0} \subseteq T$.

$A_{1} \times A_{1}: \quad H_{I}$ can have one, two, or four connected components, as $Z\left(H_{R}\right)$ $=\left\{I, \gamma_{\alpha_{1}}, \gamma_{\alpha_{2}}, \gamma_{\alpha_{1}} \gamma_{\alpha_{2}}\right\}$. However, in each case, $B=\mathfrak{h}+\sum_{\alpha \in \Phi_{R}} \delta_{S}{ }^{\alpha}$. The roots of $\left(B_{c}, \mathfrak{h}_{c}\right)$ are exactly the real roots of $\left(\mathbb{s}_{c}, \mathfrak{h}_{c}\right), W\left(\mathfrak{B}_{c}, \mathfrak{h}_{c}\right)=\left\{I, s_{\alpha_{1}}, s_{\alpha_{2}}, s_{\alpha_{1}} s_{\alpha_{2}}\right\}$, and $W_{K} \cap W\left(B_{c}, t_{c}\right)=\{I\}$. Thus if $h_{1} \in H_{I}^{*}, h_{2} \in H_{R}^{*}, h_{1} h_{2} \in H^{\prime}, h_{2}=$ $\exp \left(r H_{\alpha_{1}}^{*}+s H_{\alpha_{2}}^{*}\right)$,

$$
\begin{aligned}
\Delta_{H}\left(h_{1} h_{2}\right) \Theta_{\tau}\left(h_{1} h_{2}\right) \\
=\sum_{w \in W_{K}} \operatorname{det} w \xi_{w \tau}\left(h_{1}\right) \sum_{s \in W\left(B_{C}, \mathfrak{G} C\right)} \operatorname{det} s c_{\tau}\left(s: w: H^{*}\right) \\
\quad \times \exp \left(s^{\nu \alpha_{1}-1_{\nu_{2}}-1}(w \tau)\left(\log h_{2}\right)\right) \\
=\sum_{w \in W_{K}} \operatorname{det} w \xi_{w \tau}\left(h_{1}\right) c\left(w \tau: \mathfrak{h}^{*}\right) \exp \left(-\left.\right|^{\nu \alpha_{1}-\mathbf{1}_{\alpha_{2}}-1}(w \tau)\left(r H_{\alpha_{1}}^{*}\right) \mid\right) \\
\quad \times \exp \left(-\left.\right|^{\nu \alpha_{1}-\mathbf{1}_{\alpha_{\alpha_{2}}}-1}(w \tau)\left(s H_{\alpha_{2}}^{*}\right) \mid\right)
\end{aligned}
$$

where $\mathfrak{h}^{*}$ is the component of $\mathfrak{h}^{\prime}(R)$ corresponding to $H^{*}$. For $\mathfrak{h}^{*}=\mathfrak{h}^{+}$ $=\left\{H_{k}+r H_{\alpha_{1}}^{*}+s H_{\alpha_{2}}^{*}: H_{k} \in \mathfrak{h}_{k}, r, s>0\right\}, \tau \in L_{T}$,

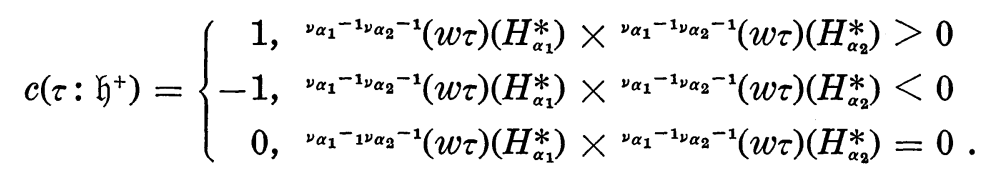

Otherwise, $c\left(\tau: s \mathfrak{h}^{+}\right)=\operatorname{det} s c\left(\tau: \mathfrak{h}^{+}\right), s \in W\left(\mathfrak{B}_{c}, \mathfrak{h}_{c}\right)$.

$B_{2}:$ If $\Phi_{R}$ is of type $B_{2}, H_{I}=H_{I}^{0} \cup \gamma_{\alpha_{1}} H_{I}^{0} \cup \gamma_{\alpha_{2}} H_{I}^{0} \cup \gamma_{\alpha_{1}} \gamma_{\alpha_{2}} H_{I}^{0}$, (the four components not necessarily distinct). The centralizer of $H_{I}^{0}$ and $\gamma_{\alpha_{2}} H_{I}^{0}$ is $3=\mathfrak{U}+\sum_{\alpha \in \Phi_{R}} \mathbb{S S}^{\alpha}$. The roots of $\left(B_{c}, \mathfrak{h}_{c}\right)$ are exactly the real roots of

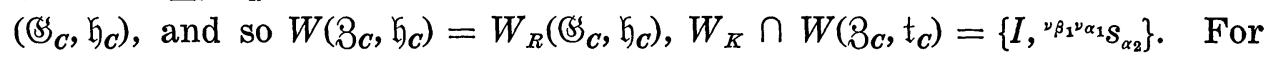
$h_{1} h_{2} \in H^{\prime}, h_{1} \in H_{I}^{0} \cup \gamma_{\alpha_{2}} H_{I}^{0}, h_{2} \in H_{R}^{*}=\exp \left(\mathfrak{h}^{*} \cap \mathfrak{p}\right), \mathfrak{h}^{*}$ a component of $\mathfrak{h}^{\prime}(R)$, we have

$$
\begin{aligned}
& \Delta_{H}\left(h_{1} h_{2}\right) \Theta_{\tau}\left(h_{1} h_{2}\right) \\
& =\sum_{w \in W K_{K} /\left\{I,{ }^{\nu} \beta_{1}{ }^{\nu} \alpha_{1} s_{\alpha_{2}}\right\}} \operatorname{det} w \xi_{w \tau}\left(h_{1}\right) \sum_{s \in W\left(\beta_{C},{ }^{\prime} C\right)} \operatorname{det} s c_{\tau}\left(s: w: H^{*}\right) \\
& \times \exp \left(s^{\nu \beta_{1}-1_{\nu \alpha_{1}}-1}(w \tau)\left(\log h_{2}\right)\right)
\end{aligned}
$$

where

$$
c_{\tau}\left(s: w: H^{*}\right)=c_{3}\left(s: w \tau: \mathfrak{h}^{*}\right)=c_{\nu \beta_{1}{ }^{-1_{\nu_{1}}-1}}\left(s: w \tau: \mathfrak{h}^{*}\right)
$$


where $c_{\nu_{\beta_{1}}-1_{\nu_{1}}{ }^{-1}}\left(s: w \tau: \mathfrak{h}^{+}\right)$is given by table (3.3) for regular $\tau$ such that $\langle\tau, \alpha\rangle \neq 0$ for $\alpha \in \Phi_{R}$. For singular $\tau$ for which $\langle\tau, \alpha\rangle=0$ for some $\alpha \epsilon$ $\Phi_{R}$, and any $w \in W\left(\mathscr{G}_{c},{ }_{c}\right) c_{\nu_{\beta_{1}}{ }^{-1} \alpha_{\alpha_{1}}{ }^{-1}}\left(w: \tau: \mathfrak{G}^{+}\right)$reduces to zero except for the

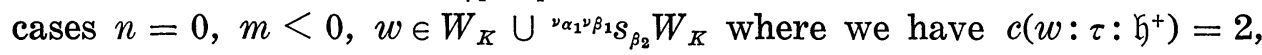
and $m=n<0, w \in W_{K} \cup{ }^{{ }^{\alpha} \nu_{1} \beta_{1}} s_{\beta_{2}} W_{K}$, where $c\left(w: \tau: \mathfrak{h}^{+}\right)=1$. $\quad(n$ and $m$ are as defined in $\S 3$ ).

The centralizer of $\gamma_{\alpha_{1}} H_{I}^{0}$ and $\gamma_{\alpha_{1}} \gamma_{\alpha_{2}} H_{I}^{0}$ is $3=\mathfrak{h}+\left(\mathscr{S}^{\alpha_{1}}+\left(\mathscr{S}^{-\alpha_{1}}+\mathscr{6}^{\beta_{1}}+\right.\right.$ $\left(\mathbb{S}^{-\beta_{1}}\right.$. The only roots of $\left(3_{c}, \mathfrak{h}_{c}\right)$ are $\pm \alpha_{1}$ and $\pm \beta_{1}$, so that the root system of 3 is of type $A_{1} \times A_{1}$ rather than of type $B_{2}$. W( $\left.3 c, \mathfrak{h}_{c}\right)=\left\{I, s_{\alpha_{1}}, s_{\beta_{1}}\right.$, $\left.s_{\alpha_{1}} s_{\beta_{1}}\right\}$, and $W_{K} \cap W\left(\S_{c}, t_{c}\right)=\{I\}$. Thus for $h_{1} h_{2} \in H^{\prime}, h_{1} \in \gamma_{\alpha_{1}} H_{I}^{0} \cup \gamma_{\alpha_{1}} \gamma_{\alpha_{2}} H_{I}^{0}$, $h_{2} \in H_{R}^{*}$,

$$
\begin{aligned}
& \Delta_{H}\left(h_{1} h_{2}\right) \Theta_{\tau}\left(h_{1} h_{2}\right) \\
& =\sum_{w \in W_{R}} \operatorname{det} w \xi_{w \tau}\left(h_{1}\right) \sum_{w \in W\left(\beta_{C},{ }^{,} c\right)} \operatorname{det} s c_{\tau}\left(s: w: H^{*}\right) \\
& \times \exp \left(s^{\nu \beta_{1}-1_{\nu_{1}}-1}(w \tau)\left(\log h_{2}\right)\right)
\end{aligned}
$$

where $c_{\tau}\left(s: w: H^{*}\right)=c_{8}\left(s: w \tau: \mathfrak{h}^{*}\right)$. In this case, since the root system of 3 is of type $A_{1} \times A_{1}$, by [2c), p. 285], we have, for $s \in W\left(3 c, \mathfrak{h}_{c}\right)$,

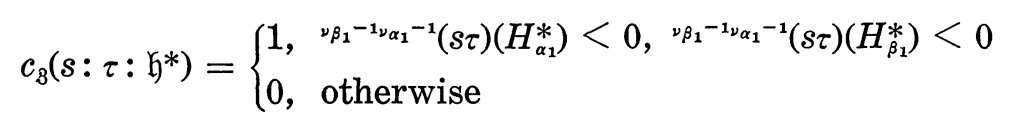

for any component $\mathfrak{h}^{*}$ of $\mathfrak{h}^{\prime}(R)$ such that $\mathfrak{h}^{*} \subseteq \mathfrak{G}_{3}^{+}=\left\{H_{k}+r H_{\alpha_{1}}^{*}+s H_{\beta_{1}}^{*}: H_{k}\right.$ $\left.\in \mathfrak{h}_{k}, r, s>0\right\}$. As in the case for $\Phi_{R}$ of type $A_{1} \times A_{1}$, the expression for $\Theta_{\tau}$ in this case simplifies to (4.3), with $\beta_{1}$ replacing $\alpha_{2}$, where $c\left(\tau: \mathfrak{h}^{*}\right)$ is defined as previously for any $\mathfrak{h}^{*} \subseteq \mathfrak{G}_{3}^{+}$. Otherwise, $c\left(\tau: \mathfrak{h}^{*}\right)=$ $c\left(^{\left({ }^{\nu}{ }_{1} \beta_{1}\right.} s \tau: s \mathfrak{h}^{*}\right)$ where $s \in W\left(\Re_{c}, \mathfrak{h}_{c}\right)$ satisfies $s \mathfrak{h}^{*} \subseteq \mathfrak{h}_{3}^{+}$.

$G_{2}:$ If $\Phi_{R}$ is of type $G_{2}, H_{I}=\{I\} \cup\left\{\gamma_{\alpha_{1}}\right\} \cup\left\{\gamma_{\alpha_{2}}\right\} \cup\left\{\gamma_{\alpha_{1}} \gamma_{\alpha_{2}}\right\}$. The centralizer of $\{I\}$ is $\mathbb{S}$, so for $h \in H_{R}^{*}$,

$$
\Delta_{H}(h) \Theta_{\tau}(h)=\sum_{s \in W\left(\beta_{B},{ }^{,} c\right)} \operatorname{det} s c_{\tau}\left(s: I: H_{R}^{*}\right) \exp \left(s^{\nu \beta_{1}-1_{\nu_{\alpha}}-1} \tau(\log h)\right)
$$

where $c_{\tau}\left(s: I: H_{R}^{*}\right)=c_{\nu \beta_{1}{ }^{-1 \alpha_{\alpha_{1}}{ }^{-1}}}\left(s: \tau: \mathfrak{h}^{*}\right)$ which for $\mathfrak{h}^{*}=\mathfrak{h}^{+}$is given in table (3.4) for regular $\tau$. For singular $\tau$, using the notation of the table, we have

$$
c\left(w: \tau: \mathfrak{h}^{+}\right)=\left\{\begin{array}{l}
4, m=n<0, w \in \in^{\nu_{\alpha_{1}{ }^{\nu} \beta_{1}}} S_{\alpha_{1}} W_{K} \\
2, m<n=0, m=-n<0,3 m=n<0, w \in \in^{\nu_{\alpha_{1}{ }^{\nu} \beta_{1}}} S_{\alpha_{1}} W_{K} \\
2,3 m=n<0, w \in W_{K}
\end{array}\right.
$$




$$
\left(\begin{array}{rl}
-2, & m<n=0, w \in W_{K} \\
2, & m=-n<0, w \in^{{ }^{\nu \alpha_{1} \beta_{1} \beta_{1}} s_{\alpha_{3}} W_{K} .}
\end{array}\right.
$$

Of course, for other components $\mathfrak{h}^{*}$, we use (2.5) together with the values of the constants for $\mathfrak{h}^{+}$.

The centralizer of $\left\{\gamma_{\alpha_{1}}\right\}$ is $3=\mathfrak{h}+\mathbb{S}^{\alpha_{1}}+\mathbb{S}^{-\alpha_{1}}+\mathbb{s}^{\beta_{1}}+\mathbb{S s}^{-\beta_{1}}$. The only roots of $\left(\mathfrak{S}_{c}, \mathfrak{h}_{c}\right)$ are $\pm \alpha_{1}$ and $\pm \beta_{1} . W\left(\mathfrak{S}_{c}, \mathfrak{h}_{c}\right)=\left\{I, s_{\alpha_{1}}, s_{\beta_{1}}, s_{\alpha_{1}} s_{\beta_{1}}\right\}$, and $W_{K}$ $\cap W\left(B_{c}, t_{c}\right)=\{I,-I\}$. Thus for $h \in H_{R}^{*}$,

$$
\begin{aligned}
& \Delta_{H}(h) \Theta_{\tau}(h) \\
& =\sum_{w \in W K /\{I,-I\}} \operatorname{det} w \xi_{w \tau}\left(\gamma_{\alpha_{1}}\right) \sum_{s \in W\left(B C, \zeta_{C}\right)} \operatorname{det} s c_{\tau}\left(s: w: H_{R}^{+}\right) \\
& \quad \times \exp \left(s^{\nu \beta_{1}^{-1}{ }^{-1} \alpha_{1}-1}(w \tau)(\log h)\right)
\end{aligned}
$$

where $c_{\tau}\left(s: w: H_{R}^{+}\right)=c_{8}\left(s: w \tau: \mathfrak{h}^{*}\right)+c_{8}\left(-s:-w \tau: \mathfrak{h}^{*}\right)$ and $c_{3}\left(s: \tau: \mathfrak{h}^{*}\right)$ is given as in (4.5). As before, the expression simplifies to (4.3) where $c\left(\tau: \mathfrak{h}^{*}\right)$ is defined as for the $B_{2}$ case, and again we replace $\alpha_{2}$ by $\beta_{1}$.

$\left\{\gamma_{\alpha_{2}}\right\} \not \subset T$, but there exist $k, k^{\prime} \in K$ such that ${ }^{k}\left(\gamma_{\alpha_{2}}\right)=\gamma_{\alpha_{1}}$ and ${ }^{k^{\prime}}\left(\gamma_{\alpha_{1}} \gamma_{\alpha_{2}}\right)$ $=\gamma_{\alpha_{1}} . \quad k$ and $k^{\prime}$ correspond to the elements $s_{\alpha_{3}}$ and $s_{\alpha_{2}}$ in $W(G, H)$ respectively. Using the invariance of $\Theta_{\tau}, \Theta_{\tau}\left(\gamma_{\alpha_{2}} h\right)=\Theta_{\tau}\left(\gamma_{\alpha_{1}} s_{\alpha_{3}} h\right)$ and $\Theta_{\tau}\left(\gamma_{\alpha_{1}} \gamma_{\alpha_{2}} h\right)$ $=\Theta_{\tau}\left(\gamma_{\alpha_{1}} s_{\alpha_{2}} h\right), h \in H_{R}^{*}$, and so can be obtained from the formulas above.

As in $\S 3$, we can give a complete description of $\Theta_{\tau}$ in the case that $G$ has exactly $n+1$ conjugacy classes of Cartan subgroups, $n=$ rank $(G / K)$. Let $H_{\ell}$ be the Cartan subgroup of $G$ corresponding to $\mathfrak{h}_{\ell}$, $0 \leq \ell \leq n$, notation as in $\S 3$.

Each component of $\left(H_{\ell}\right)_{I}$ has as centralizer in \&s, $3=\mathfrak{h}_{\ell}+\sum_{i=\ell+1}^{n}\left(\mathbb{S}^{\alpha_{i}}\right.$ $+\left(S^{-\alpha_{i}}\right)$, and $\left(3_{c}, \mathfrak{h}_{\ell c}\right)$ has roots $\pm \alpha_{\ell+1}, \cdots, \pm \alpha_{n} . W\left(B_{c}, \mathfrak{h}_{\ell c}\right)$ is the subgroup of $W\left(\mathscr{S}_{C}, \mathfrak{h}_{\ell c}\right)$ generated by the $s_{\alpha i}, i=\ell+1, \cdots, n$, and $W_{K} \cap W\left(3_{c}, t_{c}\right)$ $=\{I\}$. Thus if $h_{1} h_{2} \in H_{\ell}^{\prime}, h_{1} \in\left(H_{\ell}\right)_{I}^{*}, h_{2} \in\left(H_{\ell}\right)_{R}^{*}, h_{2}=\exp \left(\sum_{i=\ell+1}^{n} r_{i} H_{\alpha_{i}}^{*}\right)$,

$$
\begin{aligned}
\Delta_{H_{\ell}}\left(h_{1} h_{2}\right) \Theta_{\tau}\left(h_{1} h_{2}\right) \\
=\sum_{w \in W_{K}} \operatorname{det} w \xi_{w \tau}\left(h_{1}\right) \sum_{s \in W\left(B C, h_{\ell} C\right)} \operatorname{det} s c_{\tau}\left(s: w: H_{\ell}^{*}\right) \\
\quad \times \exp \left(s^{\mu \ell-1}(w \tau)\left(\log h_{2}\right)\right) \\
=\sum_{w \in W_{K}} \operatorname{det} w \xi_{w \tau}\left(h_{1}\right) c\left(w \tau: \mathfrak{h}_{\ell}^{*}\right) \exp \left(-\left.\right|^{\mu \ell-1}(w \tau)\left(r_{\ell+1} H_{\alpha_{\ell+1}}^{*}\right) \mid\right) \\
\quad \cdots \exp \left(-\left.\right|^{\mu \ell-1}(w \tau)\left(r_{n} H_{\alpha_{n}}^{*}\right) \mid\right)
\end{aligned}
$$

where for

$$
\mathfrak{h}^{*}=\mathfrak{h}^{+}=\left\{H_{k}+\sum_{i=\ell+1}^{n} r_{i} H_{\alpha_{i}}^{*}: H_{k} \in\left(\mathfrak{h}_{\ell}\right)_{k}, r_{i}>0, i=\ell+1, \cdots, n\right\},
$$




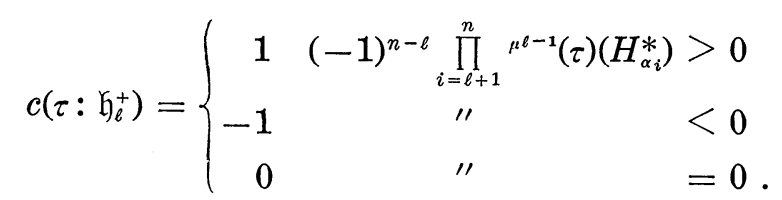

For $\mathfrak{h}^{*}=s \mathfrak{h}_{\ell}^{+}, s \in W\left(\mathfrak{B}_{c}, \mathfrak{h}_{\ell c}\right), c\left(\tau: s \mathfrak{h}^{+}\right)=\operatorname{det} s c\left(\tau: \mathfrak{h}_{\ell}^{+}\right)$.

For $\tau \in L_{T}^{\prime}$, let

$$
\varepsilon(\tau)=\operatorname{sign}\left\{\prod_{\alpha \in \Phi+(\Im) C, t C)}\langle\alpha, \tau\rangle\right\} .
$$

Let $s=\frac{1}{2} \operatorname{dim}(G / K)$. Then $T_{\tau}=(-1)^{s} \varepsilon(\tau) \Theta_{\tau}$ is the character of a discrete series representation of $G$, and all discrete series characters are of this form. $T_{\tau_{1}}=T_{\tau_{2}}$ if and only if $\tau_{1}$ and $\tau_{2}$ are conjugate by $W_{K}$.

For singular $\tau, \Theta_{\tau}$ has no known character theoretic interpretation in general. If $w \tau=\tau$ for some $w \neq 1$ in $W_{K}, \Theta_{\tau} \equiv 0$. However for other singular $\tau, \Theta_{\tau}$ need not vanish.

\section{REFERENCES}

[1] H. R. P. Ferguson, Some Integers of Harish-Chandra, Ph.D. Thesis, University of Washington, Seattle, 1971.

[2] Harish-Chandra, a) Discrete series for semisimple Lie groups. I, Acta Math. 113 (1965), 241-318.

b) Two theorems on semisimple Lie groups, Ann. of Math. (2) 83 (1966), 74-128.

c) Discrete series for semisimple Lie groups. II, Acta Math. 116 (1966), 1-111.

[ 3 ] T. Hirai, a) The Plancherel formula for SU (p,q), J. Math. Soc. Japan, 22 (1970), 134-179.

b) Explicit form of the characters of discrete series representations of semisimple Lie groups, Conference on Harmonic Analysis on Homogeneous Spaces, Williams College, Williamstown, 1972.

[ 4 ] G. Warner, Harmonic Analysis on semi-simple Lie groups, 2 volumes, SpringerVerlag, Berlin, 1972.

Institute for Advanced Study

Princeton 